\title{
Arthroscopic Long Head of the Biceps Transposition for Superior Capsular Augmentation Results in Comparable Clinical Outcomes to Arthroscopic Partial Repair for Irreparable Rotator Cuff Tears
}

\author{
Kenji Kawashima, M.D., Ph.D., Nobuo Terabayashi, M.D., Ph.D., Hiromi Asano, M.D., and \\ Haruhiko Akiyama, M.D., Ph.D.
}

Purpose: To describe a modified arthroscopic technique of long head of biceps transposition (LHBT) for superior capsular augmentation; to investigate the outcomes and effectiveness of LHBT in patients with irreparable rotator cuff tears; and to compare the results with those of arthroscopic partial repair (APR) after a 2-year minimum follow-up. Methods: We retrospectively reviewed patients who underwent arthroscopic repair of large to massive superior rotator cuff tears. The inclusion criteria were an irreparable rotator cuff with inability of the tendon to reach the original footprint and postoperative follow-up for a minimum of 2 years. We investigated 22 patients followed up for 30.7 months (mean); 10 patients underwent isolated partial repair and 12 patients underwent LHBT combined with partial repair. The acromiohumeral interval (AHI) was measured using anteroposterior radiographs. Cuff integrity was defined using Sugaya's classification 2 years postoperatively. Clinical outcomes were assessed preoperatively and during postoperative follow-up (minimum 2 years) using the American Shoulder and Elbow Surgeons and University of California, Los Angeles shoulder rating scales. Results: Postoperative functional scores significantly improved in both groups. In the LHBT group, both American Shoulder and Elbow Surgeons and University of California, Los Angeles scores significantly improved postoperatively from $52.0 \pm 14.6$ to $89.3 \pm 10.4$ points $(P=.002)$ and $15.2 \pm 2.2$ to $32.5 \pm 2.6$ points $(P=.002)$, respectively. In addition, the AHI at the final follow-up was significantly greater in the LHBT group (7.5 $\pm 2.0 \mathrm{~mm})$ than in the APR group $(5.8 \pm 1.9 \mathrm{~mm})(P=.032)$. The retear rate, forward flexion, and postoperative functional scores at the final follow-up were better in the LHBT group; however, the intergroup differences were not statistically significant. Conclusions: Arthroscopic LHBT for irreparable rotator cuff tears showed comparable clinical outcomes and improvement in postoperative AHI compared with APR after a minimum 2-year follow-up. Level of Evidence: Level III, retrospective comparative trial.

$\mathbf{R}$ ecently, superior capsular reconstruction (SCR) has been presented as a possible alternative for treating irreparable rotator cuff tears (RCTs) and restoring superior glenohumeral stability and shoulder joint function. Mihata et al. ${ }^{1}$ described an increased postoperative acromiohumeral interval (AHI) and clinical improvement after SCR. The graft used was a tensor fasciae latae (TFL) autograft that was attached

From the Department of Orthopaedic Surgery, Yamauchi Hospital (K.K.); and Gifu University Graduate School of Medicine (K.K., N.T., H.A., H.A.), Gifu, Japan.

The authors report that they have no conflicts of interest in the authorship and publication of this article. Full ICMJE author disclosure forms are available for this article online, as supplementary material.

This study was performed at Department of Orthopaedic Surgery, Gifu University Graduate School of Medicine, Gifu, Japan.

Received June 15, 2021; accepted October 24, 2021. medially to the superior glenoid and laterally to the greater tuberosity. However, there is the need for another operative procedure for TFL harvesting because of the morbidity and risk for complications associated with this treatment. In an effort to avoid TFL-associated autograft harvest-site complications, such as pain, local swelling, hematoma, numbness, and infection, the long head of the biceps (LHB) tendon has been proposed as

Address correspondence to Kenji Kawashima, M.D., Ph.D., Department of Orthopaedic Surgery, Yamauchi Hospital, 3-7-22 Ichihashi, Gifu City, 5008381, Gifu, Japan.E-mail: kawashimars@gmail.com

(C) 2021 THE AUTHORS. Published by Elsevier Inc. on behalf of the Arthroscopy Association of North America. This is an open access article under the CC BY-NC-ND license (http://creativecommons.org/licenses/by-nc-nd/4.0/).

2666-061X/21862

https://doi.org/10.1016/j.asmr.2021.10.020 
an alternative to standard SCR grafts, and several technical notes of SCR using LHB have been recently reported. $^{2-4}$ In this technique, the native LHB attachment on the glenoid side was preserved, and the proximal part of the LHB was transposed posteriorly and fixed onto the footprint as the SCR. However, only a few published reports compared the clinical results of this technique with those of other techniques. ${ }^{5}$ The purposes of this study were to describe a modified arthroscopic technique of long head of biceps transposition (LHBT) for superior capsular augmentation; to investigate the outcomes and effectiveness of LHBT in patients with irreparable rotator cuff tears; and to compare the results with those of arthroscopic partial repair (APR) after a 2-year minimum follow-up.

\section{Methods}

\section{Patient Selection}

We retrospectively investigated patients who underwent arthroscopic repair for irreparable RCTs, including the intact or repairable subscapularis tendon, between January 2013 and April 2019. Ethical approval for this study was obtained from the Gifu University School of Medicine Institutional Review Board (No. 29-316). The inclusion criteria of this study were irreparable large-tomassive RCTs with isolated APR or LHBT combined with a partial repair and a minimum of 2-year followup postoperatively. Two patients in the APR group and one in the LHBT group were excluded because their follow-up was less than 2 years. In general, we indicated APR or LHBT for operatively irreparable rotator cuff with inability of the tendon to reach the original footprint after significant releases of the capsule and subdeltoid synovial tissue to complete the repair. An isolated partial repair had been performed until 2017 and then LHBT combined with partial repair has been performed since 2018 .

A total of 22 patients (13 men and 9 women) with a mean age of 69.3 years (range, 58-79 years) underwent APR or LHBT for irreparable RCTs during the study period. Ten patients underwent APR, and 12 patients underwent arthroscopic LHBT combined with partial repair. The mean follow-up period was 30.7 months (range, 24-72 months). Details of the patient's demographics and preoperative clinical data are presented in Table 1.

\section{Radiographic Evaluation}

Standardized anteroposterior radiographs of the shoulder, which are routine for patients with shoulder problems, were used for the evaluation of the AHI. The AHI, the closest distance between the humeral head and the undersurface of the acromion, was measured using the preoperative and final postoperative anteroposterior radiographs of the patients in standing
Table 1. Patient Demographics and Preoperative Clinical Data

\begin{tabular}{|c|c|c|c|}
\hline & $\begin{array}{l}\text { APR Group } \\
(\mathrm{n}=10)\end{array}$ & $\begin{array}{l}\text { LHBT Group } \\
\quad(\mathrm{n}=12)\end{array}$ & $P$ Value \\
\hline Age, $y^{\dagger}$ & $71.9 \pm 7.5$ & $67.8 \pm 2.0$ & .22 \\
\hline Mean follow-up (range), mo & $37.2(24-72)$ & $24.8(24-30)$ & \\
\hline Sex (male:female) & $6: 4$ & $7: 5$ & \\
\hline AHI, $\mathrm{mm}^{*}$ & $7.7 \pm 2.8$ & $6.8 \pm 1.8$ & .67 \\
\hline \multicolumn{4}{|l|}{ Hamada grade } \\
\hline I & 6 & 8 & \\
\hline II & 3 & 4 & \\
\hline III & 1 & & \\
\hline \multicolumn{4}{|l|}{ Size } \\
\hline Large & 2 & 4 & \\
\hline Massive & 8 & 8 & \\
\hline GFDI $^{*}$ & $2.33 \pm 0.70$ & $2.14 \pm 0.25$ & .69 \\
\hline \multicolumn{4}{|l|}{ Preoperative $\mathrm{ROM}^{\dagger}$} \\
\hline $\mathrm{FF},{ }^{\circ}$ & $116 \pm 40$ & $108 \pm 47$ & .69 \\
\hline $\mathrm{ER}, \circ$ & $37 \pm 20$ & $44 \pm 19$ & .24 \\
\hline $\mathrm{IR},^{\circ}$ & $\mathrm{Ll} \pm 4$ & $\mathrm{~T} 11 \pm 3$ & .32 \\
\hline \multicolumn{4}{|l|}{ Preoperative clinical score ${ }^{*}$} \\
\hline ASES & $53.5 \pm 14.1$ & $52.0 \pm 14.6$ & .36 \\
\hline UCLA & $16.1 \pm 2.8$ & $15.1 \pm 2.2$ & .35 \\
\hline
\end{tabular}

AHI, acromiohumeral interval; APR, arthroscopic partial repair; ASES, American Shoulder and Elbow Surgeons; ER, external rotation; FF, forward flexion; GFDI, Goutallier fatty degeneration index; IR, internal rotation behind the back; LHBT, long head of biceps transposition; ROM, range of motion; UCLA, the University of California, Los Angeles.

*Mann-Whitney $U$ test.

${ }^{\dagger}$ Data are presented as means \pm standard deviation.

position to evaluate superior migration of the humeral head. Rotator cuff tear-related shoulder arthritis was evaluated on plain preoperative radiograph using the Hamada classification. ${ }^{6}$ All shoulders were assessed by an experienced shoulder surgeon (H.A.) in a blind fashion.

\section{Magnetic Resonance Imaging (MRI) Evaluation}

MRI was performed with a 1.5-T system (Vantage Titan 1.5T; Toshiba Medical Systems, Otawara, Japan). Preoperative fatty degeneration was evaluated for each muscle using the Goutallier classification on oblique sagittal T2-weighted MRIs. ${ }^{7}$ The global fatty degeneration index, which corresponds to the mean value of the Goutallier stages for the subscapularis (SSC), supraspinatus (SSP), and infraspinatus (ISP) muscles, was calculated for each shoulder. ${ }^{8}$ Repaired cuff integrity was evaluated using Sugaya's classification ${ }^{9}$ on oblique coronal T2-weighted MRIs 2 years after surgery, and type $\mathrm{V}$ (the presence of a major discontinuity) was considered as a retear. Although types IV and V were commonly considered as retears in most studies, type IV was not considered as such in this study, because a minor discontinuity of the repaired superior rotator cuff often remained after partial repair. Transposed LHB grafts in the LHBT group also were evaluated on oblique coronal T2-weighted MRIs. We used a slice that showed the attachment of the LHB. Discontinuity of the 
Fig 1. Grade of long head of the biceps (LHB) lesions. Intra-articular view from the posterior portal in the right shoulders, with the patients in the beach-chair position. The LHB lesions are classified into 4 grades (6 subgrades) and assessed by arthroscopic findings. LHB is divided into 4 equal parts, and the extent of the partial tendon tear, which is focal fiber rupture, is assessed. Longitudinal tears are excluded from the partial tears. Grade 0: normal; grade Ia: redness, flattening, fraying, or longitudinal split; grade Ib: $<25 \%$ partial tear; grade Ic: $25 \%$ to $50 \%$ partial tear; grade II: $>50 \%$ partial tear; grade III: complete tear.
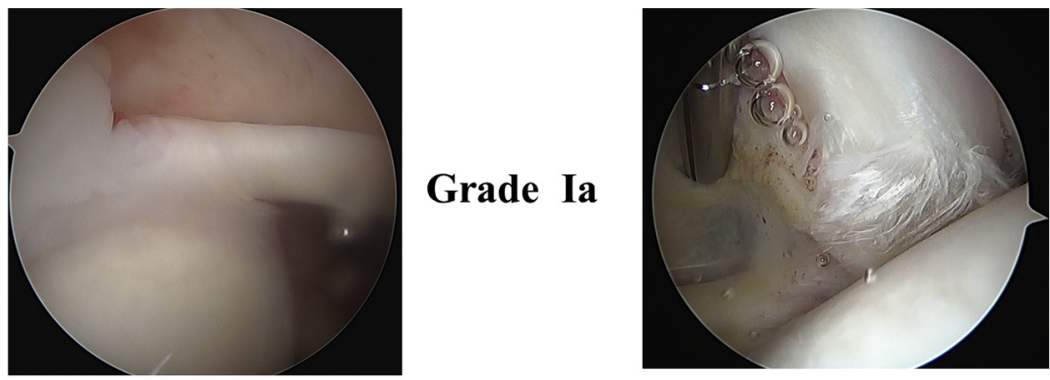

Grade Ib
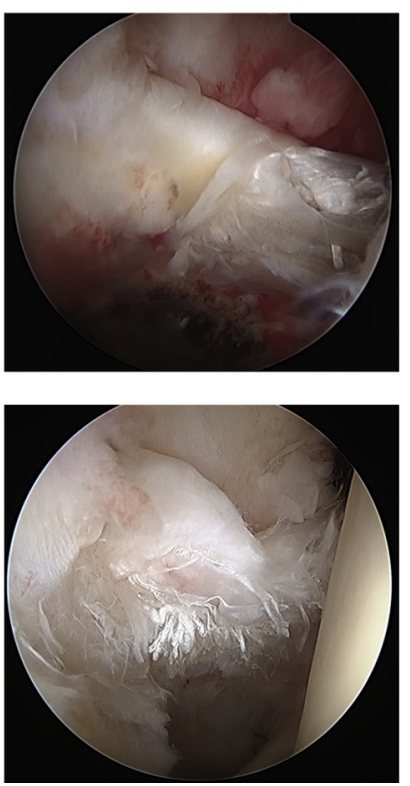

Grade Ic

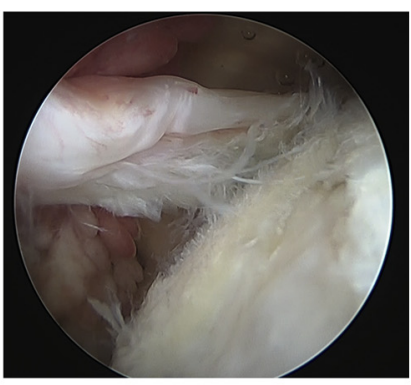

Grade III transposed LHB was defined as graft failure. The MRI parameters of the T2-weighted oblique coronal scans were as follows: repetition time 3,500 milliseconds; echo time 94 milliseconds; field of view $200 \mathrm{~mm}$; and matrix $512 \times 800$. For the T2-weighted oblique sagittal scans, the parameters were: repetition time 4,400 milliseconds; repetition time 94 milliseconds; field of view $200 \mathrm{~mm}$; and matrix $224 \times 352$.

\section{Surgical Procedure}

\section{Diagnostic Arthroscopy}

Surgeries were performed by 2 experienced shoulder surgeons (K.K. and N.T.) with the patient under general anesthesia and in the beach-chair position with an interscalene block. The glenohumeral joint, articular side of the SSP tendon, SSC tendon, and LHB were viewed through the posterior portal and evaluated. We modified Lafosse's grade $^{10}$ of LHB lesions based on arthroscopic findings and divided grade I into 3 subtypes. The quality of the LHB was graded as follows: 0, intact LHB; Ia, minor lesions with redness, flattening, fraying or longitudinal split of the LHB; Ib, < 25\% partial tear; Ic, $25 \%$ to $50 \%$ partial tear; and II, $>50 \%$ partial tear; III, complete tear. Details of the grades are presented in Fig 1.

\section{Mobilization}

After diagnostic arthroscopy, subacromial decompression was performed. Both the articular and subacromial sides of the SSP and ISP tendons were mobilized, and degenerative tendons were debrided. After significant releases of the capsule, coracohumeral ligament, and subdeltoid synovial tissue, tear size was evaluated as the mediolateral width according to the classification of DeOrio and Cofield $^{11}$ : small, $<1 \mathrm{~cm}$; medium, 1 to $3 \mathrm{~cm}$; large, 3 to $5 \mathrm{~cm}$; and massive, $>5$ $\mathrm{cm}$. Then, rotator cuffs in which the tendon could not reach the lateral edge of the normal footprint were diagnosed as irreparable RCTs, thus indicated for LHBT combined with partial repair (Fig 2).

\section{Long Head of Biceps Transposition}

The transverse humeral ligament was released, and the LHB was removed from the bicipital groove. A no. 2 nylon suture was used to grasp, control, and check the mobility of the LHB. By pulling the nylon suture posteriorly, the appropriate position of the LHB shift at the superior facet of the greater tuberosity was confirmed. Two anchors with triple-loaded sutures were used for dual-row fixation. The medial anchor was placed at the edge of the articular cartilage medial to the highest 

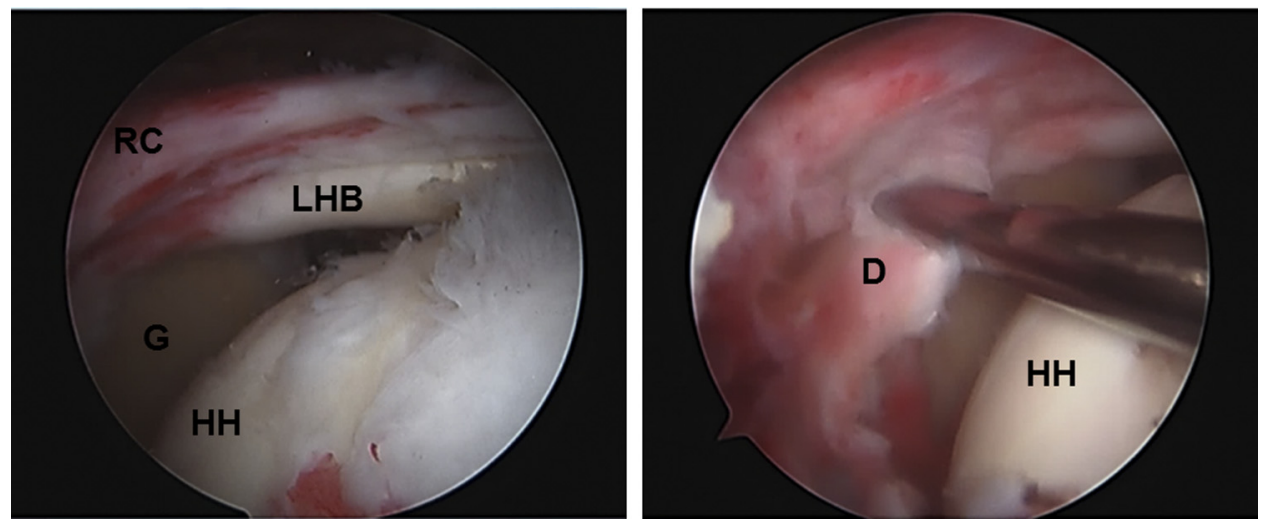

Fig 2. Arthroscopic view from the lateral portal in a right shoulder, with the patient in the beachchair position, showing a massive rotator cuff with inability of the supraspinatus and infraspinatus tendons to reach the original footprint after routine release of the capsule and subdeltoid synovial tissue. (D, deep layer of rotator cuff; G, glenoid; $\mathrm{HH}$, humeral head; LHB, long head of the biceps; $\mathrm{RC}$, rotator cuff.)

point of the greater tuberosity (Fig 3A). Two different sutures of the medial anchor were passed through the LHB with lasso-loop stitch, ${ }^{12}$ and the end of another suture was passed under the LHB using IDEAL Suture Grasper $60^{\circ}$ (DePuy Mitek, Raynham, MA) (Fig 3B). The lateral anchor was placed 5 to $10 \mathrm{~mm}$ posterior to the bicipital groove at the superior facet (Fig 3C), and the same suture management as the medial anchor was performed, with the sutures tied using half-hitch knots for lasso-loop stitch, and another suture tied using a sliding knot to secure the LHB at $30^{\circ}$ of shoulder abduction and $15^{\circ}$ of external rotation (Fig 3D).

\section{Suprapectoral LHB Tenodesis}

After the proximal part of the LHB was secured in a new location, suprapectoral LHB tenodesis was performed. The triple-loaded suture anchor was inserted just proximal to the insertion of the pectoralis major at the bicipital groove. The sutures were passed through the LHB using a lasso-loop technique, as previously described. Before tying the suture, LHB was transected using radiofrequency distal to the lateral anchor used for LHBT at the superior facet. Then, the distal part of the tenotomized LHB was fixed to the proximal humerus by tying the remaining sutures for suprapectoral biceps tenodesis.
Fig 3. Long head of the biceps (LHB) transposition procedures. Arthroscopic view from the lateral portal in the right shoulder, with the patient in the beach-chair position. (A) The medial row anchor is placed at the edge of the articular cartilage medial to the highest point of the greater tuberosity. (B) Suture passage through the LHB with lasso-loop stitch (arrow) using a retrograde suture retriever. (C) The lateral anchor is inserted 5 to $10 \mathrm{~mm}$ posterior to the bicipital groove at the superior facet. (D) The sutures are tied and LHB is secured onto the superior aspect of the greater tuberosity at $30^{\circ}$ of shoulder abduction and $15^{\circ}$ of external rotation. (G, glenoid; $\mathrm{HH}$, humeral head; LHB, long head of the biceps; $\mathrm{RC}$, rotator cuff.)
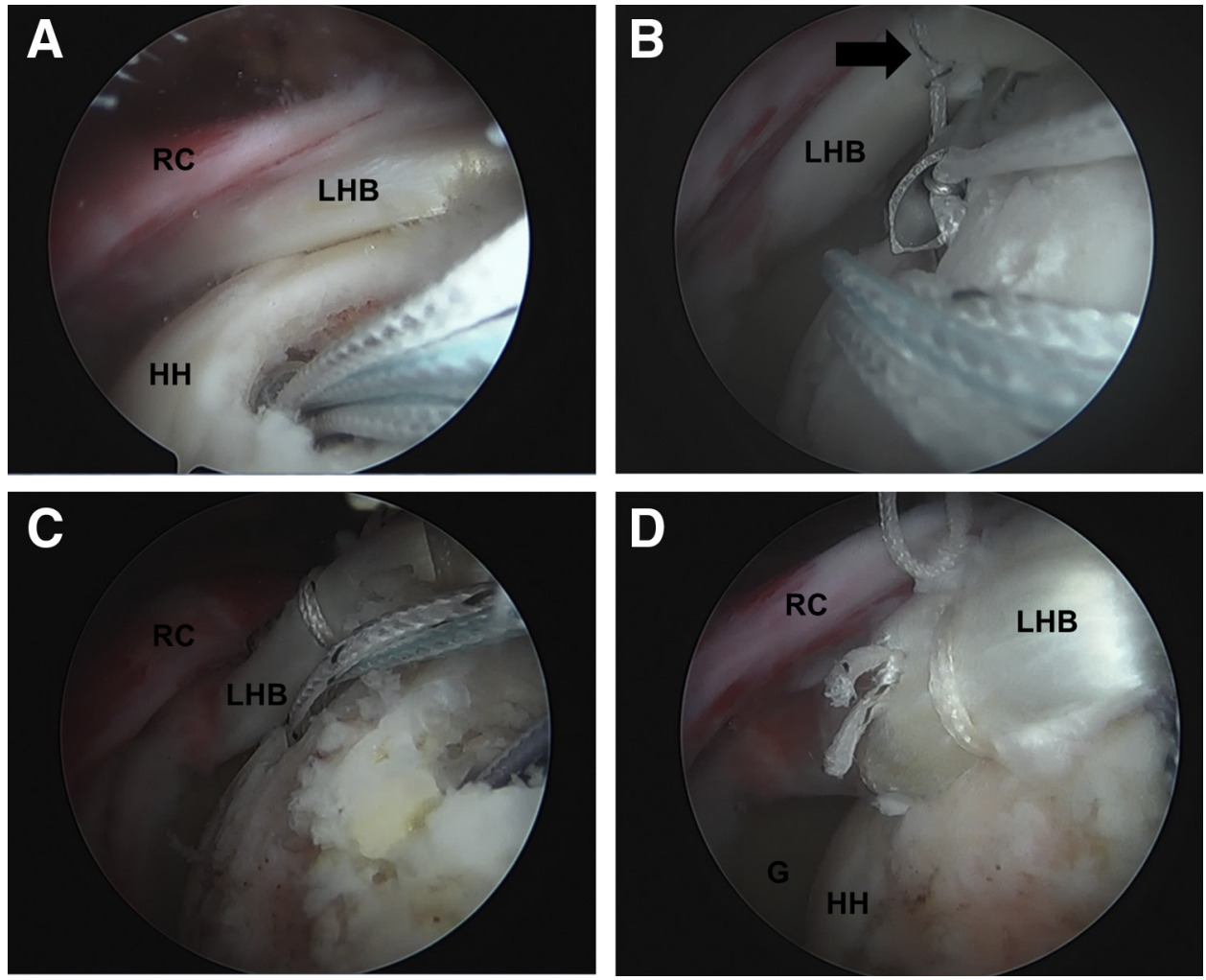
Fig 4. Partial repair of posterior -superior rotator cuff. Arthroscopic view from the lateral portal in the right shoulder, with the patient in the beach-chair position. (A) The triple-loaded anchor for posterior-superior rotator cuff repair is inserted at the slightly medialized footprint posterior to the transposed long head of the biceps (LHB). (B) Suture passage through the rotator cuff tendon. (C) The posterior-superior rotator cuff is secured using a single-row technique. (D) A microfracture awl is used to penetrate the subchondral bone on the footprint immediately lateral to the fixation points with suture anchors as a bone marrowstimulating technique. (FP, footprint; G, glenoid; $\mathrm{HH}$, humeral head; LHB, long head of the biceps; RC, posterior-superior rotator cuff.)
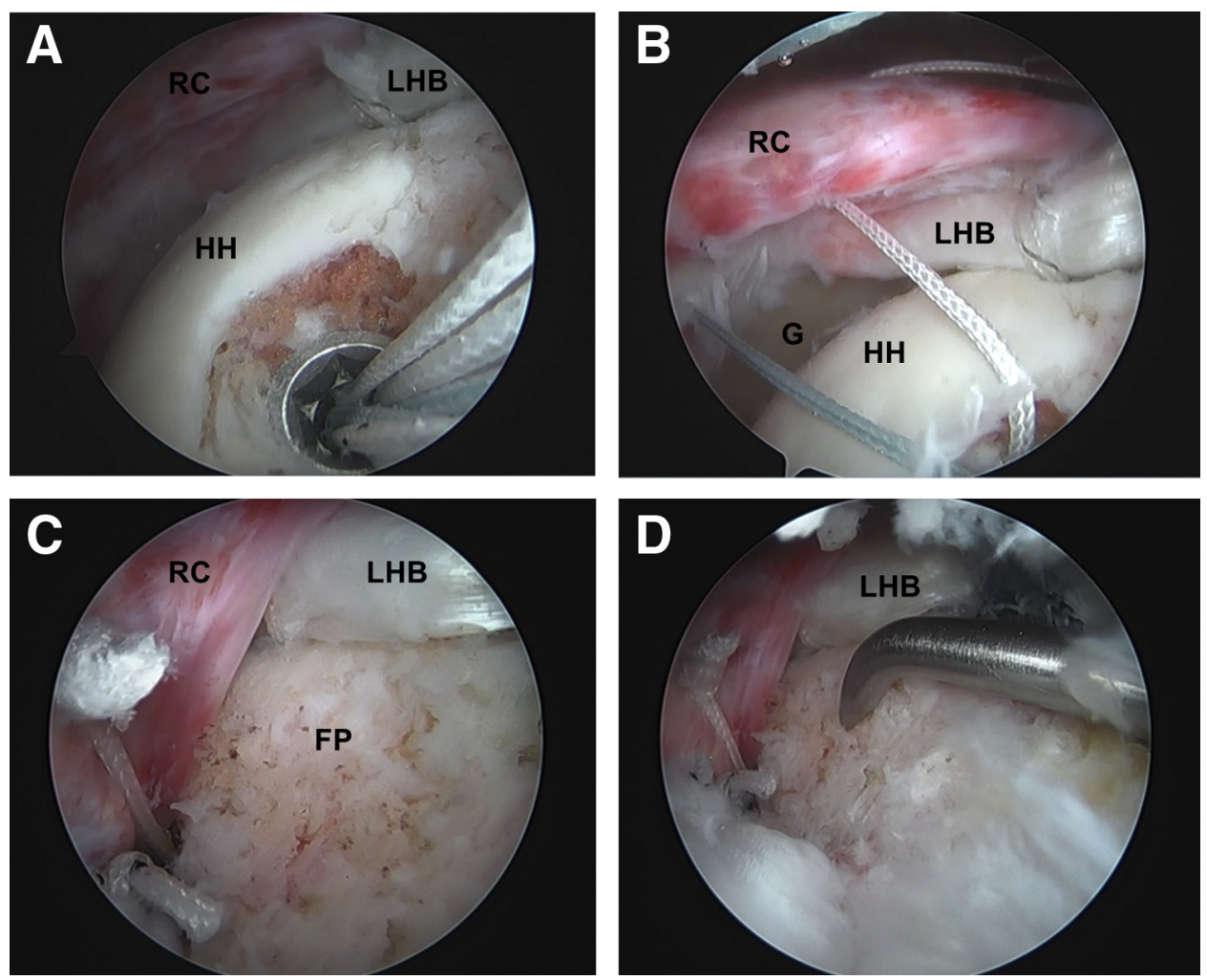

\section{SSC Tendon Repair}

Repair of full-thickness SSC tears was performed using the single-row or suture-bridge technique ${ }^{13,14}$ according to the tear size.

\section{Partial Repair of Superior Rotator Cuff}

After LHBT, down-migration of the humeral head was often achieved, making it easier to repair the retracted rotator cuff tendon without excessive tension. The triple-loaded suture anchor for anterior-superior rotator cuff repair was placed at a slightly medialized footprint anterior to the transposed LHB. The sutures were then passed through the anterior-superior rotator cuff, and the knot was tied for single-row fixation. An additional anchor for posterior-superior rotator cuff repair was inserted at the slightly medialized footprint posterior to the transposed LHB (Fig 4A). Then, the posterior-superior rotator cuff was secured in the same way as the anterior-superior rotator cuff (Fig 4B-C). Side-to-side suturing between the transposed LHB and repaired anterior or posterior rotator cuff tendons was not performed to prevent undue tension on the graft. Finally, microfracture as a bone marrow-stimulating technique $^{15}$ was performed on the footprint immediately lateral to the fixation points with suture anchors to enhance tendon-to-bone healing (Fig 4D).

\section{Postoperative Rehabilitation}

Shoulders were immobilized using an abduction sling for 5 weeks after surgery. Isometric rotator cuff and relaxation exercises of the shoulder girdle muscles were initiated immediately after surgery. Passive and activeassisted range of motion (ROM) exercises were initiated after sling removal, and active ROM and muscle strengthening exercises were initiated 6 weeks after surgery. Light physical activity or exercise was allowed 3 months after surgery, and heavy labor or sports activities were allowed 6 months after surgery, according to each patient's functional recovery.

\section{Patient Assessment}

Each patient's active ROM (forward flexion, external rotation at the side, and internal rotation behind the back) was pre- and postoperatively measured with a goniometer by one of our experienced shoulder surgeons (K.K. or N.T.). Shoulder function was also assessed using the American Shoulder and Elbow Surgeons (ASES) and the University of California, Los Angeles (UCLA) shoulder rating scales. Clinical outcome measures were evaluated preoperatively and postoperatively at the final follow-up. Complications and reoperations were reviewed from the medical records. 
Table 2. Comparison of Preoperative and Postoperative Data in Each Group

\begin{tabular}{|c|c|c|c|}
\hline & Pre & Final & $P$ Value \\
\hline \multicolumn{4}{|l|}{ APR group } \\
\hline \multicolumn{4}{|l|}{ ROM } \\
\hline 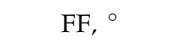 & $116 \pm 40$ & $134 \pm 44$ & .23 \\
\hline $\mathrm{ER},{ }^{\circ}$ & $37 \pm 20$ & $40 \pm 17$ & .50 \\
\hline $\mathrm{IR},^{\circ}$ & $\mathrm{Ll} \pm 4$ & $\mathrm{~T} 11 \pm 2$ & .28 \\
\hline \multicolumn{4}{|c|}{ Clinical score } \\
\hline ASES & $53.5 \pm 14.1$ & $83.4 \pm 10.1$ & .006 \\
\hline UCLA & $16.1 \pm 2.8$ & $29.8 \pm 4.7$ & .006 \\
\hline AHI, mm & $7.7 \pm 2.8$ & $5.8 \pm 1.9$ & .08 \\
\hline \multicolumn{4}{|l|}{ LHBT group } \\
\hline \multicolumn{4}{|l|}{ ROM } \\
\hline $\mathrm{FF},{ }^{\circ}$ & $108 \pm 47$ & $160 \pm 13$ & .01 \\
\hline $\mathrm{ER},{ }^{\circ}$ & $44 \pm 19$ & $40 \pm 19$ & .59 \\
\hline $\mathrm{IR}^{\circ}{ }^{\circ}$ & $\mathrm{T} 11 \pm 3$ & $\mathrm{~T} 10 \pm 2$ & .09 \\
\hline \multicolumn{4}{|l|}{ Clinical score } \\
\hline ASES & $52.0 \pm 14.6$ & $89.3 \pm 10.4$ & .002 \\
\hline UCLA & $15.1 \pm 2.2$ & $32.5 \pm 2.6$ & .002 \\
\hline AHI, mm & $6.8 \pm 1.8$ & $7.5 \pm 2.0$ & .29 \\
\hline
\end{tabular}

NOTE. Data are presented as means \pm standard deviation.

AHI, acromiohumeral interval; APR, arthroscopic partial repair; ASES, American Shoulder and Elbow Surgeons; ER, external rotation; FF, forward flexion; IR, internal rotation behind the back; LHBT, long head of biceps transposition; ROM, range of motion; UCLA, the University of California. Los Angeles.

*Wilcoxon signed rank test.

\section{Statistical Analysis}

Statistical differences between the 2 groups were analyzed using the Mann-Whitney $U$ test. The Fisher exact test was used to compare retear rates. Preoperative and postoperative clinical scores were compared using the Wilcoxon signed-rank test. All analyses were performed using GraphPad Prism 8.0 software (GraphPad Software, Inc., San Diego, CA), and $P$ values $<.05$ were considered statistically significant.

\section{Results}

\section{Acromiohumeral Interval}

In the APR group, the AHI measured on anteroposterior shoulder radiographs decreased from $7.7 \pm$ $2.8 \mathrm{~mm}$ before surgery to $5.8 \pm 1.9 \mathrm{~mm}$ at final followup without statistical significance $(P=.08)$. In the LHB group, the AHI increased from $6.8 \pm 1.8 \mathrm{~mm}$ before surgery to $7.5 \pm 2.0 \mathrm{~mm}$ at final follow-up without statistical significance $(P=.29)$ (Table 2). However, the intergroup difference in AHI at the final follow-up point showed statistical significance $(P=.032)$ (Table 3$)$, indicating that the LHBT group maintained a postoperative AHI better than the APR group.

\section{Repaired Cuff Integrity}

The retear rates based on MRI 2 years after surgery were $80 \%$ in the APR group and $42 \%$ in the LHBT group. The LHBT group showed better outcomes in terms of the retear rates; however, the difference was not statistically significant (odds ratio, 0.18; $P=.099$ ) (Table 3). In the LHBT group, graft failure was observed in 5 patients $(42 \%)$. All 5 patients with graft failure showed retear of the rotator cuff repair on postoperative MRI, whereas all 7 patients without graft failure did not have retear. Patients with $<25 \%$ partial tears of LHB (grades 0 to Ib in our classification) had neither graft failure nor retear.

\section{Clinical Outcomes}

In the APR group, no statistically significant changes were observed in shoulder forward flexion (from $116^{\circ} \pm 40^{\circ}$ to $134^{\circ} \pm 44^{\circ}, P=.23$ ), external rotation at the side (from $37^{\circ} \pm 20^{\circ}$ to $40^{\circ} \pm 17^{\circ}, P=.50$ ), and internal rotation behind the back (from $\mathrm{Ll} \pm 4$ to $\mathrm{Tl} l \pm$ $2, \mathrm{P}=0.28$ )

In the LHB group, shoulder forward flexion improved significantly (from $108^{\circ} \pm 47^{\circ}$ to $160^{\circ} \pm 13^{\circ}, P=.013$ ); however, no statistically significant changes were observed in external rotation at the side (from $44^{\circ} \pm$ $19^{\circ}$ to $\left.40^{\circ} \pm 19^{\circ}, P=0.59\right)$ and internal rotation behind the back (from Tll \pm 3 to Tlo $\pm 2, P=.085$ ) (Table 2 ).

Postoperative forward flexion was greater in the LHBT group, although it did not reach statistical significance $(P=.20)$ (Table 3$)$. In the APR group, the mean ASES score significantly improved from $53.5 \pm 14.1$ points (range, $16-68$ points) to $83.4 \pm 10.1$ points (range, 68 -100 points; $P=.006$ ), and the mean UCLA score also improved from $16.1 \pm 2.8$ points (range, $12-22$ points) to $29.8 \pm 4.7$ points (range, $21-35$ points; $P=.006$ ). In the LHBT group, the mean ASES score significantly improved from $52.0 \pm 14.6$ points (range, 19-80 points) to $89.3 \pm 10.4$ points (range, $73-100$ points; $P=.002$ ), and the mean UCLA score also improved from $15.1 \pm$ 2.2 points (range, $13-21$ points) to $32.5 \pm 2.6$ points (range, 26-35; $P=.002$ ) (Table 2). Both the ASES scores $(P=.21)$ and UCLA scores $(P=.19)$ at the final

Table 3. Comparison of the Final Outcomes Between the 2 Groups

\begin{tabular}{lccc}
\hline & APR & LHBT & $P$ Value \\
\hline ROM $^{*}$ & & & \\
FF, $^{\circ}$ & $134 \pm 44$ & $160 \pm 13$ & $.20^{\dagger}$ \\
ER, $^{\circ}$ & $40 \pm 17$ & $40 \pm 19$ & $.81^{\dagger}$ \\
IR, $^{\circ}$ & T11 \pm 2 & T10 & \\
Clinical score & & & $.42^{\dagger}$ \\
ASES & $83.4 \pm 10.1$ & $89.3 \pm 10.4$ & $.21^{\dagger}$ \\
UCLA & $29.8 \pm 4.7$ & $32.5 \pm 2.6$ & $.19^{\dagger}$ \\
AHI, mm & $5.8 \pm 1.9$ & $7.5 \pm 2.0$ & $.032^{\dagger}$ \\
Retear rate, n $(\%)$ & $8 / 10(80)$ & $5 / 12(42)$ & $.099^{\ddagger}$ \\
\hline
\end{tabular}

AHI, acromiohumeral interval; APR, arthroscopic partial repair; ASES, American Shoulder and Elbow Surgeons; ER, external rotation; $\mathrm{FF}$, forward flexion; IR, internal rotation behind the back; LHBT, long head of biceps transposition; ROM, range of motion; UCLA, the University of California, Los Angeles.

*Data are presented as means \pm standard deviation.

${ }^{\dagger}$ Mann-Whitney $U$ test.

${ }^{\ddagger}$ Fisher exact test. 
Table 4. Number of Patients Meeting the Threshold for Minimal Clinically Important Difference, Substantial Clinical Benefit, and Patient Acceptable Symptomatic State ${ }^{16}$ for Each Procedure

\begin{tabular}{ccc}
\hline Threshold $^{16}$ & APR Group $(\mathrm{n}=10)$ & LHBT Group $(\mathrm{n}=12)$ \\
\hline ASES score & & \\
MCID $(21.0)$ & $8 / 10$ & $10 / 12$ \\
SCB $(26.0)$ & $7 / 10$ & $9 / 12$ \\
PASS $(78.0)$ & $7 / 10$ & $10 / 12$ \\
UCLA score & & \\
MCID (6.0) & $9 / 10$ & $12 / 12$ \\
SCB $(8.0)$ & $8 / 10$ & $9 / 12$ \\
PASS $(23.0)$ & $9 / 10$ & $12 / 12$ \\
\hline
\end{tabular}

ASES, American Shoulder and Elbow Surgeons; LHBT, long head of biceps transposition; MCID, minimal clinically important difference; PASS, patient acceptable symptomatic state; SCB, substantial clinical benefit; UCLA, the University of California, Los Angeles.

follow-up were better in the LHBT group than in the APR group; however, the intergroup differences were not statistically significant (Table 3 ).

One patient in the APR group required reverse shoulder arthroplasty 3 years postoperatively, because of loss of shoulder function and persistent pain. In contrast, one patient in the LHBT group required reoperation because of graft failure with early suture anchor pullout. Neither infection nor abnormal inflammatory reactions were observed in any of the patients postoperatively.

In terms of clinically meaningful outcomes, number of patients meeting the threshold for minimal clinically important difference, substantial clinical benefit, and patient acceptable symptomatic state ${ }^{16}$ for each procedure is listed in Table 4.

\section{Discussion}

Our LHBT technique demonstrated good functional and radiologic outcomes after a 2-year minimum follow-up. Postoperative functional scores significantly improved compared with the preoperative scores. The LHBT group maintained the AHI better than the APR group at the final follow-up. Thus, arthroscopic LHBT for superior capsular augmentation could be an option for surgical treatment of irreparable RCTs.

Previous studies have described various LHB augmentation techniques and good clinical results for massive RCTs. ${ }^{17-20}$ In some studies, LHB was used as a free graft to augment rotator cuff repairs for massive RCTs. ${ }^{19,21-23}$ Sano et al. ${ }^{19}$ reported that the LHB tendon patch grafting method provided significant improvement in both the active elevation angle and the Japanese Orthopaedic Association score. In other studies, LHB tenotomy was performed at the insertion site of the tendon at the border of the glenoid, and the tenotomized LHB was attached to the greater tuberosity in the rotator cuff footprint. ${ }^{20,24-26}$ Cho et al. ${ }^{20}$ reported that the arthroscopic augmentation technique using a tenotomized biceps tendon was effective in achieving fewer structural failures and equivalent Constant and UCLA scores in comparison with the traditional arthroscopic repair techniques.

Recently, SCR using a TFL autograft has been introduced as an effective procedure for retaining the static stability of the shoulder joint and preventing progression to cuff tear arthropathy (CTA). Several studies have indicated an increased AHI postoperatively and clinical improvement in patients with irreparable massive RCTs after rotator cuff repair with SCR using a TFL autograft. ${ }^{27,28}$ Along with the popularization of SCR, there have been several technical reports of SCR using proximal LHB, in which the native LHB attachment on the glenoid side was preserved and the LHB was shifted to its new location on the greater tuberosity. ${ }^{2-4,29-31}$ Although the LHB was not as wide as the TFL, it was observed that the transposed LHB could potentially serve as the superior capsule. This technique was called "biological SCR," ${ }^{2}$ "partial SCR," ${ }^{3}$ or "modified SCR as reinforcement." ${ }^{4}$ There have been several biomechanical studies on SCR using LHB. ${ }^{32,33}$ Using a cadaveric rotator cuff tear model, Han et al. ${ }^{32}$ found that shoulders in which the biceps were used for SCR showed decreased subacromial peak contact pressures and shifted the humeral head inferiorly. In addition, El-Shaar et al. ${ }^{33}$ described that SCR using the

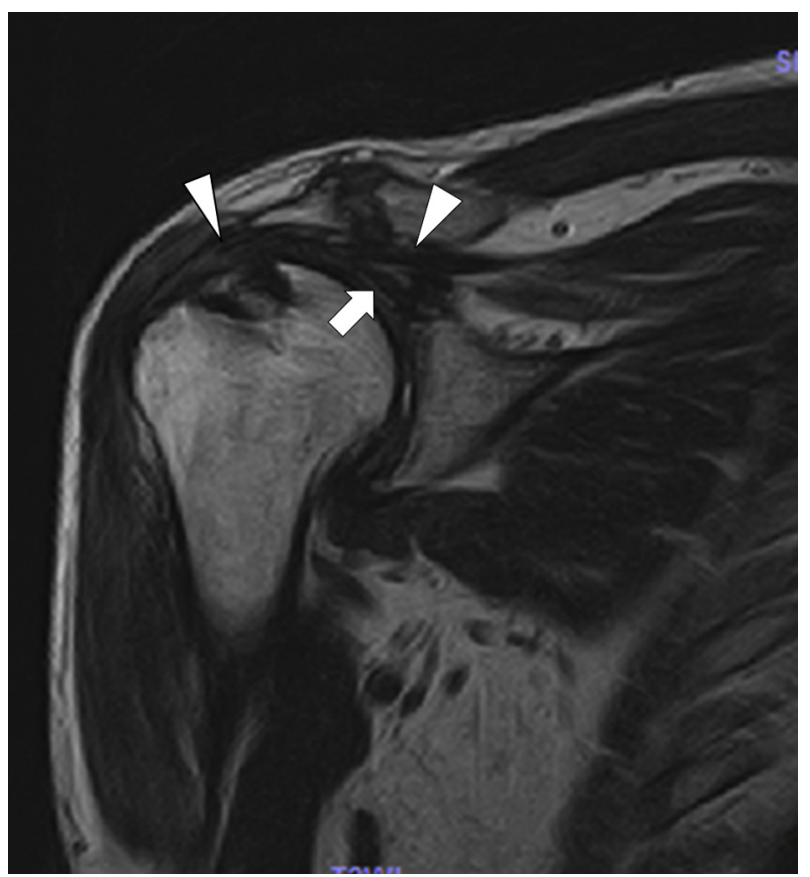

Fig 5. Postoperative $\mathrm{T} 2$-weighted oblique coronal magnetic resonance image of right shoulder in the 70-year-old male patient 2 years after arthroscopic long head of the biceps (LHB) transposition. The transposed LHB (arrow) remains attached at the superior facet. The repaired superior rotator cuff (arrowhead) covers the transposed LHB. 
LHB was biomechanically equivalent to using a TFL autograft by analyzing matched cadaveric shoulders. These biomechanical studies showed that SCR using the LHB appeared to provide an effective downward force to the humeral head, increasing postoperative AHI. A decrease in AHI was associated with re-tear of RCTs and increased risk of CTA in other studies. ${ }^{34,35}$ In the past, we were not satisfied with a decreased postoperative AHI with the conventional APR method. The patients after APR occasionally had persistent pain with subacromial impingement. We found that it was important to increase AHI to prevent retear and CTA. Thus, we have switched surgical techniques and added LHBT for superior capsular augmentation since 2018.In this article, we describe our modified surgical techniques that use LHB for superior capsular augmentation in patients with irreparable RCTs. The LHBT technique has several advantages. First, this technique potentially appears to provide an effective downward force to the humeral head, reducing superior head migration and making it easier to repair the retracted rotator cuff tendon without excessive tension. Partial repair of the rotator cuff with static ligamentous support for superior capsular augmentation may help maintain a congruent glenohumeral joint. Second, the blood supply to the proximal part of the LHB, mainly coming from the anterodorsal surface of the tendon, ${ }^{36}$ may be maintained, compared with that of the free autograft, because the native LHB attachment on the glenoid side is preserved. Thus, this technique may enhance the healing potential of rotator cuff repair and reduce retear rates. Third, donor-site morbidity associated with TFL autografts is avoided. LHB tenodesis is one of the surgical methods used to address LHB lesions, which are commonly associated with RCTs. In the LHBT procedure, tenotomized proximal LHB can be used more effectively as a local autograft for superior capsular augmentation without the need for additional graft harvesting. Lastly, this technique is much less technically demanding, and the operation time may be reduced compared with SCR using a TFL autograft.

However, the LHBT technique has several disadvantages. First, this technique may cause side effects such as pain related to loading of the LHB, including a SLAP lesion, which has not been previously reported. In our case, all patients in the LHBT group had type I SLAP lesions according to diagnostic arthroscopy, and there were no complaints postoperatively associated with the SLAP lesions. Further research is needed to determine whether these side effects may occur. Second, this technique cannot be used for completely torn or severely damaged LHB. Previous studies have described that their technique could not be used in patients with more than $20 \%$ to $30 \%$ partial tear of the biceps tendon. ${ }^{3,4}$ However, we were unable to find the basis of these reports, and it remained unclear how much damage to LHB was acceptable. Thus, it is important to establish indication criteria for LHBT. Lafosse et al. ${ }^{10}$ created an arthroscopic grade of LHB lesions, which was classified as normal (grade 0), having a minor lesion (grade I), or having a major lesion (grade II). We further classified grade I into 3 subtypes to determine the indications for LHBT. In this retrospective study, graft failure was observed in 5 patients $(42 \%)$ with LHB lesions grades Ic to II. Unfortunately, all 5 patients showed retear of the rotator cuff repair on postoperative MRI. In contrast, transposed LHB with grade 0 to $\mathrm{Ib}$ lesions in 7 patients $(58 \%)$ was well fixed at postoperative follow-up after 2 years (Fig 5). Moreover, retear of the rotator cuff was not observed in any of the 7 patients. These results suggest that preventing graft failure is key to achieving better clinical outcomes. The procedure can be an option for irreparable RCTs as long as the LHB is not severely damaged. Therefore, we recommend that LHBT should be used in patients with $<25 \%$ partial tears of LHB (grades 0 to Ib in our classification). If the LHB is partially torn by more than $25 \%$ (grades Ic and II) or completely torn (grade III lesion), another treatment option should be considered. Additional research is needed to validate these findings.

To avoid graft failure and improve clinical results after arthroscopic LHBT, it is important to prevent overload of the transposed LHB. Thus, unlike that described in previous technical notes, side-to-side suturing between the transposed LHB and repaired anterior or posterior rotator cuff tendon was not performed. ${ }^{29,37,38}$ Furthermore, it is necessary to evaluate the proper shoulder position for LHB fixation to avoid undue tension.

\section{Limitations}

This study has several limitations. First, this was a retrospective study with a mid-term follow-up, different lengths of the follow-ups, and it lacked multiple follow-up time points. In addition, the number of patients was small; a study with a larger number of patients is required to better appreciate the differences between groups. Second, we compared the results with only partial repair and not with other augmentation techniques such as SCR using a TFL or allograft. Another limitation is that, in the case of a severely torn LHB, alternative techniques, including SCR using a TFL or allograft, are indicated.

\section{Conclusions}

Arthroscopic LHBT for irreparable rotator cuff tears showed comparable clinical outcomes and improvement in postoperative AHI compared with APR after a minimum 2-year follow-up.

\section{References}

1. Mihata T, McGarry MH, Pirolo JM, Kinoshita M, Lee TQ. Superior capsule reconstruction to restore superior 
stability in irreparable rotator cuff tears: A biomechanical cadaveric study. Am J Sports Med 2012;40:2248-2255.

2. Adrian SC, Field LD. Biceps transposition for biological superior capsular reconstruction. Arthrosc Tech 2020;9: e841-e846.

3. Kim DS, Yeom J, Park J, Cha J. L-shape superior capsular augmentation technique using biceps tendon: The biceps LShape shifting technique. Arthrosc Tech 2020;9:e703-e709.

4. Chiang $\mathrm{CH}$, Shaw L, Chih WH, Yeh ML, Su WR. Arthroscopic rotator cuff repair combined with modified superior capsule reconstruction as reinforcement by the long head of the biceps. Arthrosc Tech 2019;8:e1223-e1231.

5. Kocaoglu B, Firatli G, Ulku TK. Partial rotator cuff repair with superior capsular reconstruction using the biceps tendon is as effective as superior capsular reconstruction using a tensor fasciae latae autograft in the treatment of irreparable massive rotator cuff tears. Orthop J Sports Med 2020;8:2325967120922526.

6. Hamada K, Yamanaka K, Uchiyama Y, Mikasa T, Mikasa M. A radiographic classification of massive rotator cuff tear arthritis. Clin Orthop Relat Res 201 1;469:2452-2460.

7. Goutallier D, Postel JM, Bernageau J, Lavau L, Voisin MC. Fatty muscle degeneration in cuff ruptures. Pre- and postoperative evaluation by CT scan. Clin Orthop Relat Res 1994:78-83.

8. Goutallier D, Postel J-M, Gleyze P, Leguilloux P, Van Driessche S. Influence of cuff muscle fatty degeneration on anatomic and functional outcomes after simple suture of full-thickness tears. J Shoulder Elbow Surg 2003;1 2:550-554.

9. Sugaya H, Maeda K, Matsuki K, Moriishi J. Repair integrity and functional outcome after arthroscopic double-row rotator cuff repair. A prospective outcome study. J Bone Joint Surg Am 2007;89:953-960.

10. Lafosse L, Reiland Y, Baier GP, Toussaint B, Jost B. Anterior and posterior instability of the long head of the biceps tendon in rotator cuff tears: A new classification based on arthroscopic observations. Arthroscopy 2007;23:73-80.

11. DeOrio JK, Cofield RH. Results of a second attempt at surgical repair of a failed initial rotator-cuff repair. J Bone Joint Surg Am 1984;66:563-567.

12. Lafosse L, Van Raebroeckx A, Brzoska R. A new technique to improve tissue grip: "The lasso-loop stitch." Arthroscopy 2006;22:1246.el-e3.

13. Kawashima K, Sugaya H, Takahashi N, et al. Relationship between the morphology of the greater tuberosity and radiological and clinical outcomes after arthroscopic rotator cuff repair. JSES Int 2021;5:493-499.

14. Shibayama K, Sugaya H, Matsuki K, et al. Repair integrity and functional outcomes after arthroscopic suture bridge subscapularis tendon repair. Arthroscopy 2018;34: 2541-2548.

15. Bilsel K, Yildiz F, Kapicioglu M, et al. Efficacy of bone marrow-stimulating technique in rotator cuff repair. J Shoulder Elbow Surg 2017;26:1360-1366.

16. Kim DM, Kim TH, Kholinne E, et al. Minimal clinically important difference, substantial clinical benefit, and patient acceptable symptomatic state after arthroscopic rotator cuff repair. Am J Sports Med 2020;48:2650-2659.

17. Nobuhara K, Hata Y, Komai M. Surgical procedure and results of repair of massive tears of the rotator cuff. Clin Orthop Relat Res 1994;304:54-59.
18. Hermanowicz K, Góralczyk A, Malinowski K, Jancewicz P, Domżalski ME. Long head biceps tendonnatural patch for massive irreparable rotator cuff tears. Arthrosc Tech 2018;7:e473-e478.

19. Sano H, Mineta M, Kita A, Itoi E. Tendon patch grafting using the long head of the biceps for irreparable massive rotator cuff tears. J Orthop Sci 2010;15:310-316.

20. Cho NS, Yi JW, Rhee YG. Arthroscopic biceps augmentation for avoiding undue tension in repair of massive rotator cuff tears. J Shoulder Elbow Surg 2009;25:183-191.

21. Neviaser JS. Ruptures of the rotator cuff of the shoulder. New concepts in the diagnosis and operative treatment of chronic ruptures. Arch Surg 1971;102:483-485.

22. Obma PR. Free biceps tendon autograft to augment arthroscopic rotator cuff repair. Arthrosc Tech 2013;2: e441-e445.

23. Veen EJD, Koorevaar CT, Diercks RL. Using the long head of biceps tendon autograft as an anatomical reconstruction of the rotator cable: An arthroscopic technique for patients with massive rotator cuff tears. Arthrosc Tech 2018;7:e699-e703.

24. Checchia SL, Doneux PS, Miyazaki AN, et al. Biceps tenodesis associated with arthroscopic repair of rotator cuff tears. J Shoulder Elbow Surg 2005;14:138-144.

25. Park SR, Sun DH, Kim J, Lee HJ, Kim JB, Kim YS. Is augmentation with the long head of the biceps tendon helpful in arthroscopic treatment of irreparable rotator cuff tears? J Shoulder Elbow Surg 2018;27:1969-1977.

26. Chen RE, Bakhsh WR, Lipof JS, McVicker ZG, Voloshin I. Rotator cuff anterior cable reconstruction with long head of biceps tendon autograft. Arthrosc Tech 2020;9:e711-e715.

27. Mihata T, Lee TQ, Watanabe C, et al. Clinical results of arthroscopic superior capsule reconstruction for irreparable rotator cuff tears. Arthroscopy 2013;29:459-470.

28. Sochacki KR, McCulloch PC, Lintner DM, Harris JD. Superior capsular reconstruction for massive rotator cuff tear leads to significant improvement in range of motion and clinical outcomes: A systematic review. Arthroscopy 2019;35:1269-1277.

29. Boutsiadis A, Chen S, Jiang C, Lenoir H, Delsol P, Barth J. Long head of the biceps as a suitable available local tissue autograft for superior capsular reconstruction: "The Chinese Way." Arthrosc Tech 2017;6:e1559-e1566.

30. Kim YS, Lee HJ, Park I, Sung GY, Kim DJ, Kim JH. Arthroscopic in situ superior capsular reconstruction using the long head of the biceps tendon. Arthrosc Tech 2018;7:e97-e103.

31. Milano G, Marchi G, Bertoni G, et al. Augmented repair of large to massive delaminated rotator cuff tears with autologous long head of the biceps tendon graft: The arthroscopic "cuff-plus" technique. Arthrosc Tech 2020;9: e1683-e1688.

32. Han F, Kong CH, Hasan MY, Ramruttun AK, Kumar VP. Superior capsular reconstruction for irreparable supraspinatus tendon tears using the long head of biceps: A biomechanical study on cadavers. Orthop Traumatol Surg Res 2019;105:257-263.

33. El-Shaar R, Soin S, Nicandri G, Maloney M, Voloshin I. Superior capsular reconstruction with a long head of the biceps tendon autograft. A cadaveric study. Orthop J Sports Med 2018;6:2325967118785365. 
34. Kuptniratsaikul V, Laohathaimongkol T, Umprai V, Yeekian C, Prasathaporn N. Pre-operative factors correlated with arthroscopic reparability of large-to-massive rotator cuff tears. BMC Musculoskelet Disord 2019;20:111.

35. Factors associated with the development of early- to midterm cuff-tear arthropathy following arthroscopic rotator cuff repair. J Shoulder Elbow Surg 2021;30:1572-1580.

36. Phornphutkul C, Tahwang S, Settakorn J. Effects of type II SLAP lesion repair techniques on the vascular supply of the long head of the biceps tendon: A cadaveric injection study. J Shoulder Elbow Surg 2021;30:772-778.

37. Chillemi C, Mantovani M, Gigante A. Superior capsular reconstruction of the shoulder: The ABC (Arthroscopic Biceps Chillemi) technique. Eur J Orthop Surg Traumatol 2018;28:1215-1223.

38. Giacomo AFD, Park MC, Lee TQ. Anterior cable reconstruction using the proximal biceps tendon for large rotator cuff defects. Arthrosc Tech 2021;10:e807-e813. 